\title{
Phase-Matched Self-Doubling Optical Parametric Oscillator
}

\author{
Tolga Kartaloğlu, Kahraman G. Köprülü, Orhan Aytür \\ Department of Electrical and Electronics Engineering, Bilkent University \\ TR-06533 Bilkent, Ankara, Turkey
}

Telephone: 90-312-266-4307 Fax: 90-312-266-4126 e-mail: aytur@ee.bilkent.edu.tr

Frequency conversion using synchronously pumped optical parametric oscillators (OPO) extend the wavelength range of ultrafast laser systems [1]. Further wavelength extension can be obtained by frequency doubling one of the OPO beams with the use of another crystal inside the cavity [2].

We report a self-doubling OPO where a single nonlinear crystal is employed for both parametric generation and frequency doubling. Our self-doubling OPO is based on a KTP $\left(\mathrm{KTiOPO}_{4}\right)$ crystal that is pumped by a Ti:Sapphire laser operating at a wavelength of $739 \mathrm{~nm}$. The KTP crystal is cut such that the signal wavelength of the OPO is at $1064 \mathrm{~nm}$, corresponding to an idler wavelength of $2420 \mathrm{~nm}$. The OPO cavity resonates only the signal wavelength. The signal beam is also phase-matched for second harmonic generation (SHG) at the same crystal orientation. With proper polarization rotation, an output beam at a wavelength of $532 \mathrm{~nm}$ can be obtained. To our knowledge, this is the first demonstration of optical parametric oscillation and phase-matched frequency doubling within a single crystal.

We use an $8 \mathrm{~mm}$ long KTP crystal cut for doubling $1064 \mathrm{~nm}$ in a type-II phase-matching geometry $\left(\theta=90^{\circ}, \phi=23^{\circ}\right)$. The crystal has antireflection coatings for the fundamental and second-harmonic wavelengths. The second-harmonic output is an extraordinary wave polarized in the horizontal ( $y$-axis) direction. In parametric generation, the pump at $739 \mathrm{~nm}$ and the signal at $1064 \mathrm{~nm}$ are both horizontally polarized, and the idler at $2420 \mathrm{~nm}$ is vertically ( $z$-axis) polarized.

A mode-locked Ti:Sapphire laser (Coherent, Mira 900F) with approximately $100 \mathrm{fs}$ long pulses at a repetition rate of $76 \mathrm{MHz}$ provides the pump beam to the OPO at a wavelength of $739 \mathrm{~nm}$. We constructed a ring cavity consisting of four mirrors that are high reflectors at $1064 \mathrm{~nm}$ as shown in Figure 1. M1 and M2 are $25 \mathrm{~cm}$ radius concave, M3 is a $3 \mathrm{~m}$ radius concave, and M4 is a flat mirror. The KTP crystal is positioned at the intracavity focus between M1 and M2. The pump beam is focused with a lens (L) of focal length $15 \mathrm{~cm}$ and enters into the cavity through M1. For efficient frequency doubling, a half-wave retarder at $1064 \mathrm{~nm}$ is placed inside the OPO cavity which couples some of the horizontally polarized signal beam to the vertical. The length of the cavity is adjusted by moving M4 in order to synchronize the intracavity signal pulses with the pump pulses. The frequency doubled beam at $532 \mathrm{~nm}$ exits the cavity through M2. In addition, there is a weak $1064 \mathrm{~nm}$ beam

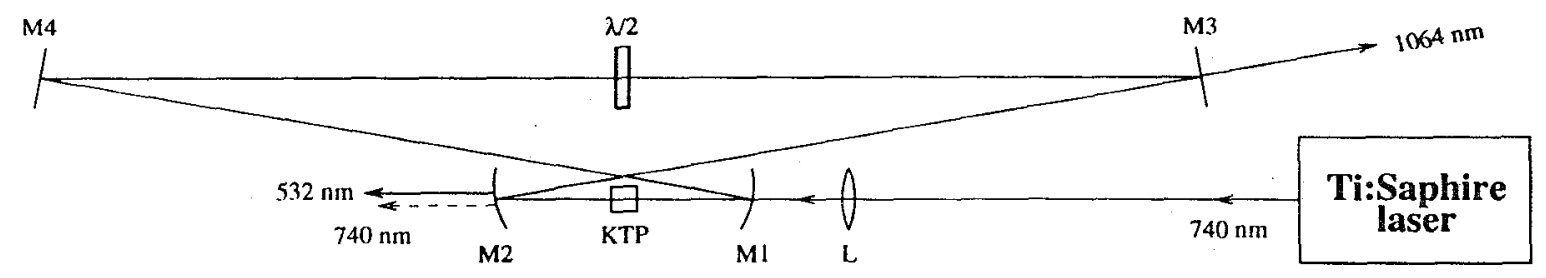

Figure 1: Self-doubling OPO setup. 
coming out through M3, as this mirror has a slightly lower reflectance compared to the other cavity mirrors. This output is used to probe the signal beam for measurements.

When the half-wave retarder is not in the cavity, there is no (or very little) frequency doubling. This results in an undercoupled OPO cavity for the signal beam. We measured the threshold for this OPO to be $60 \mathrm{~mW}$. At a pump power of $500 \mathrm{~mW}$, the pump beam is depleted by more than $50 \%$, showing strong conversion. In the case of frequency doubling with the retarder in place, the threshold of the OPO becomes $170 \mathrm{~mW}$. At a pump power of $440 \mathrm{~mW}, 25 \mathrm{~mW}$ of $532 \mathrm{~nm}$ is obtained, corresponding to $6 \%$ conversion efficiency to the green. Figure 2 shows the pump depletion with the retarder in the cavity. Depletion is seen to be as high as before, indicating strong conversion to the signal wavelength. The autocorrelation width of the green pulses are measured to be $18 \mathrm{ps}$. This pulse broadening is due to group velocity mismatch in the crystal and the retarder.

We expect significant improvement on these initial results by optimizing the cavity mode, crystal length, and using prisms to compensate for the group velocity mismatch. To this end, we designed a self-doubling OPO which uses a non-critically phase-matched KTP crystal. In this OPO, when the pump beam is at $744 \mathrm{~nm}$, the OPO signal is at $1080 \mathrm{~nm}$, and the doubled signal is at $540 \mathrm{~nm}$. It is possible to tune this self-doubling OPO by changing the wavelength of the pump beam together with the crystal angles. Varying $\theta$ from $90^{\circ}$ to $75^{\circ}\left(\phi=0^{\circ}\right)$ and the pump wavelength from $744 \mathrm{~nm}$ to $750 \mathrm{~nm}$, it is possible to generate frequency doubled output between $540 \mathrm{~nm}$ and $564 \mathrm{~nm}$. Changing $\phi$ from $0^{\circ}$ to $30^{\circ}\left(\theta=90^{\circ}\right)$ and the pump wavelength from $745 \mathrm{~nm}$ to $739 \mathrm{~nm}$, one can get $540 \mathrm{~nm}$ to $529 \mathrm{~nm}$ frequency doubled output.

This work was supported in part by Tubitak under Grant No. EEEAG-118 and NATO under Grant No. TU-MIMIC.

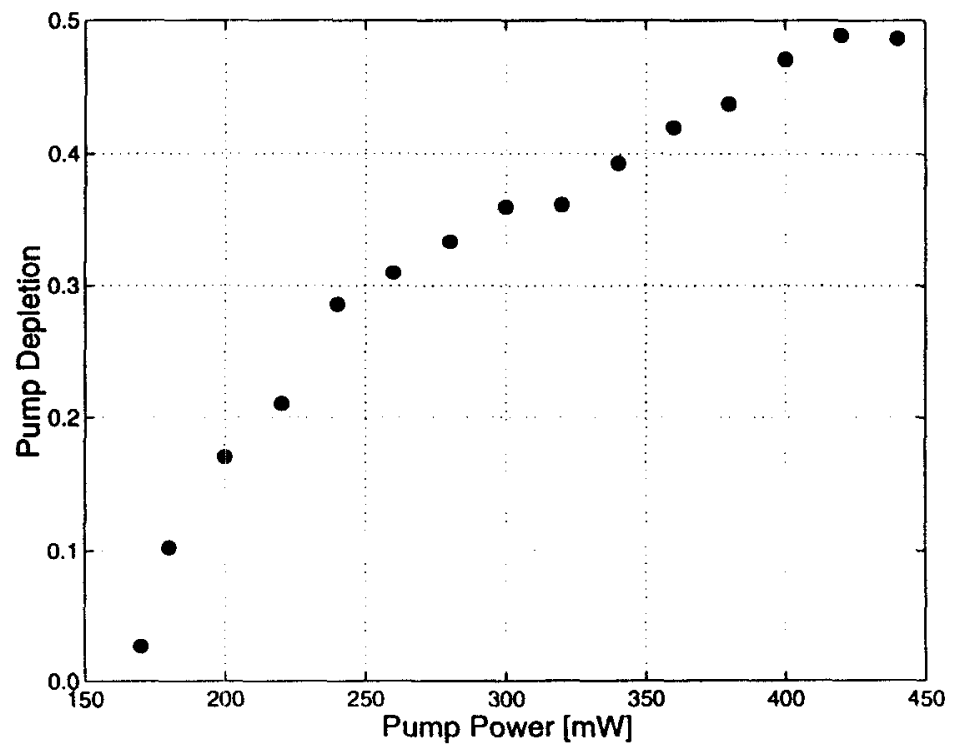

Figure 2: Pump depletion as a funcion of pump power.

[1] Special issue on Optical Parametric Oscillators and Amplifiers, J. Opt. Soc. Am. B, vol. 10, No. 11, pp. 1656-2243, 1993.

[2] P.E. Powers, R.J. Ellingson, W.S. Pelouch, and C.L.Tang, J. Opt. Soc. Am. B, vol. 10, pp. 2162-2167, 1993. 